\title{
Numerical study on the effects of aspect ratio of two types of fin folds on the propulsion performance by fish larvae's swimming simulation
}

\author{
Tatsuya BAMBA**, Tomohiro FUKUI* and Koji MORINISHI* \\ * Department of Mechanical Engineering, Kyoto Institute of Technology \\ Matsugasaki Goshokaido-cho, Sakyo-ku, Kyoto 606-8585, Japan \\ E-mail: fukui@kit.ac.jp \\ ** Department of Master's Program of Mechanophysics, Kyoto Institute of Technology \\ Matsugasaki Goshokaido-cho, Sakyo-ku, Kyoto 606-8585, Japan
}

Received: 13 January 2021; Revised: 14 June 2021; Accepted: 28 June 2021

\begin{abstract}
For clarification of an ecological system or application to ship propulsion mechanisms, studies of fish swimming have been conducted. However, many parts remain unexplained due to complexity and diversity of the swimming mechanism. In this study, we focused on the shape change of the fish larvae's fin fold and investigated effects of aspect ratio of fish larvae's fin fold on its propulsion performance by numerical simulations. Flow around the fish model was simulated by the regularized lattice Boltzmann method. To describe curved boundary on a Cartesian grid, the virtual flux method was applied. 4-tired multi block method was employed, and calculation domain was divided into 4 blocks with different grid resolutions. In this simulation, aspect ratio was defined by the ratio of fin fold length to fin fold width at the tail end, and fish models with different aspect ratio of fin fold were applied under constant surface area condition. As a result, vortices were generated along the fish body, and edge vortices pairs rotating in the opposite direction were observed at the fin fold. The edge vortices contributed to generate thrust force by increasing the front-back pressure difference at the fin fold. Compared to lower aspect ratio models, higher aspect ratio models generated more edge vortices pairs during a cycle. Accordingly, at lower aspect ratio, thrust force became larger by the wider fin fold, but amplitude of swimming speed changes in a cycle also became larger. In contrast, higher aspect ratio models swam efficiently by stabilizing its swimming speed.
\end{abstract}

Keywords : Biomimetics, Fish larvae, Fin fold, Self-propulsion, Cyclic swimming, Edge vortex

\section{Introduction}

Recently, bioinspired swimming robots are used in various situations. An example of these robots is Autonomous Underwater Vehicles (AUVs). AUV is a type of robot designed for ocean investigation (Maki, 2018). Most of AUVs are propelled by the screw propeller, which is a propulsion system for ships. However, it is still necessary to improve their performance due to occurrence of cavitation and generation of swirling flow. Cavitation degrades the propulsive performance of AUVs, and swirling flow makes an unnatural flow, which leads to destruction of the ecological system. Therefore, in recent years, movement of swimming fish has attracted attention to AUVs' new propulsion system, because they swim efficiently and suitably under severe water environment. Besides, another example of bioinspired robots is small medical robots: self-propelled endoscope (Ota et al., 2015) and 3D-printed fish for applications ranging from directed drug delivery to detoxification (Wei et al., 2015). Compared to the previous medical robots, these robots have advantages such as reducing load to the human body and high operability.

Reasons for focusing on fish swimming are that fish has outstanding characteristics including high efficiency, high turning performance and water environmental suitability. On the other hand, however, fish propulsion mechanism is quite complicated. Due to the buoyancy force balanced with fish weight, they cover much wider range in swimming 
speed and body size, and type of swimming motion varies greatly depending on their growth process and habitat. For instance, concerned with the growth process of zebrafish, they operate in the viscous flow regime at early larval stage, and it shifts from viscous flow regime to intermediate flow regime and then to inertial flow regime as they grow (Mchenry and Lauder, 2005). Hence, the swimming mechanism has not been completely clarified yet.

In recent research, studies on hydrodynamic complex phenomena and model close to live fish were reported by applying computational fluid dynamics: hydrodynamic interactions between fins, stability of a fish school and effect of fin flexibility (Liu et al., 2017; Li et al., 2019; Luo et al., 2020). On the contrary, based on the results obtained from biological experiments and numerical simulations, several bioinspired fish robots were developed. Aritani et al. (2019) designed COMET, which is a fish-like robot that can automatically track a live goldfish. They reported that further improvement of the turning performance is needed for autonomous tracking of another species of fish. In addition, Zhu et al. (2019) developed Tunabot based on yellowfin tuna. Tunabot operates at high frequencies and swims faster than the previous fish robots. They surmised that improvements of the multi-fin system and fin flexibility lead to increase swimming performance and result in acquiring more fish-like kinematic patterns.

Many researchers focused on fast swimming fish such as tuna, however, fish larvae or microorganisms are not paid much attention. Li et al. (2016) investigated vortex structure and swimming performance for fish larvae's swimming by three-dimensional numerical simulations. They clarified that the edge vortices generated along the continuous fin (fin fold) are effective in improving the propulsive performance for the wide range of flow fields with Reynolds number 14 $<R e<5500$. The shape of fish larvae's fin fold changes depending on their growth stages, and it may be optimized for surrounding environment. In other words, fin fold shape of the fish larva might be optimized to support its swimming efficiency. On the other hand, thrust force or posture control may depend on size of the fin fold: larger fin fold is more effective. Therefore, it is important to seek more effective shapes of the fin fold under the same area condition in order to design better AUVs. In this research, shapes of the fin fold including rectangle and trapezoid were defined using aspect ratio under the same area condition. As a fundamental research for the propulsion mechanism, we focused on the relation between propulsion performance and aspect ratio of fin fold. The propulsion performance was evaluated by swimming speed, thrust force, power requirement and swimming efficiency.

\section{Nomenclature}

$\begin{array}{ll}f_{\alpha} & \text { distribution function } \\ f_{\alpha}^{e q} & \text { equilibrium distribution function } \\ f_{\alpha}{ }^{n e q} & \text { non-equilibrium distribution function } \\ p_{\alpha} & \text { pressure distribution function } \\ p_{\alpha}{ }^{e q} & \text { equilibrium pressure distribution function } \\ p_{\alpha}{ }^{n e q} & \text { non-equilibrium pressure distribution function } \\ \Pi_{i j}{ }^{n e q} & \text { non-equilibrium stress tensor } \\ \boldsymbol{e}_{\alpha} & \text { discrete velocity vector } \\ w_{\alpha} & \text { weight factor } \\ c & \text { speed of advection } \\ c_{S} & \text { speed of sound } \\ \tau & \text { relaxation time } \\ \delta_{i j} & \text { Kronecker delta } \\ \delta x & \text { lattice size } \\ \delta t & \text { time step size } \\ \rho_{0} & \text { fluid reference density } \\ \rho & \text { fluid density } \\ p & \text { pressure } \\ \boldsymbol{u} & \text { fluid velocity } \\ L & \text { characteristic length } \\ U & \text { characteristic velocity } \\ R e & \text { Reynolds number } \\ v & \text { fluid kinematic viscosity } \\ A R & \text { aspect ratio } \\ & \end{array}$




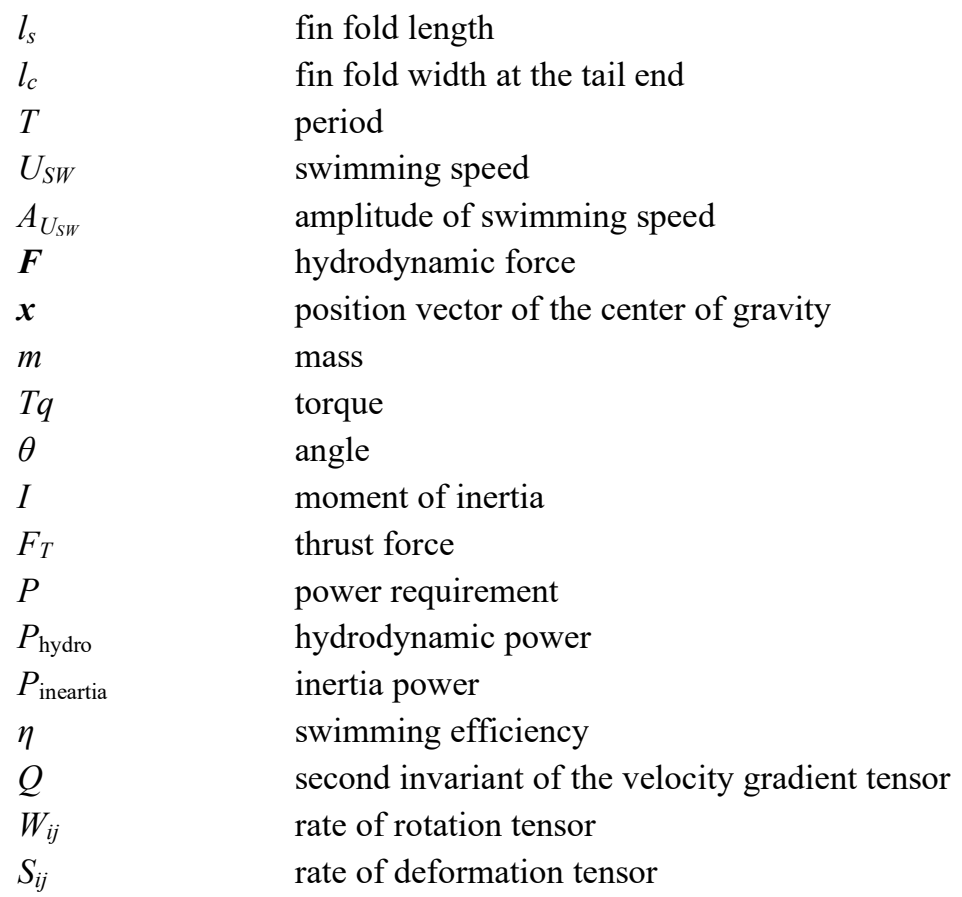

\section{Numerical method}

\subsection{Fish model and body dynamics}

The fish body models were formed by an ellipsoid based NACA0012 wing. Figure 1 shows front and bird's eye views for the longest fin fold model and description of two types of fin folds: rectangle and trapezoid fin folds. Note that origin of the coordinate is set on the tip of the fish head. Aspect ratio $A R$ was defined by the ratio of fin fold length $l_{s}$ to fin fold width at the tail end $l_{c}$.

$$
A R=\frac{l_{s}}{l_{c}} .
$$

As shown in the shaded area in Fig. 1(a), net fin fold area was set constant among the models, i.e., fin fold area does not include the fish body area.

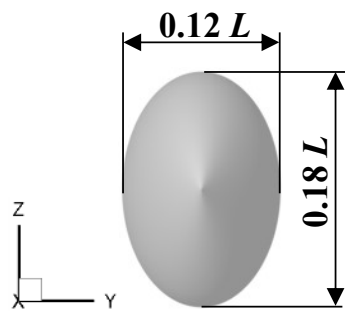

Front view

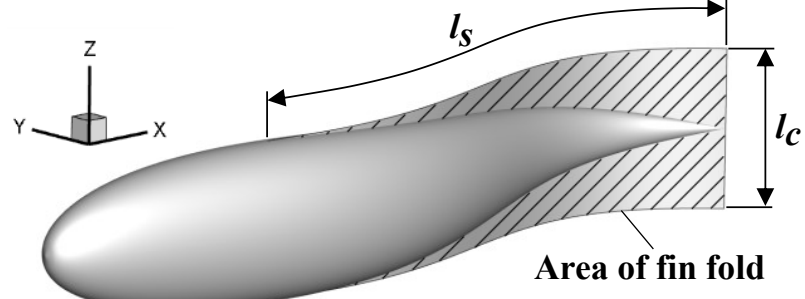

Bird's eye view

(a) $A R=3.93$ (The longest fin fold model)

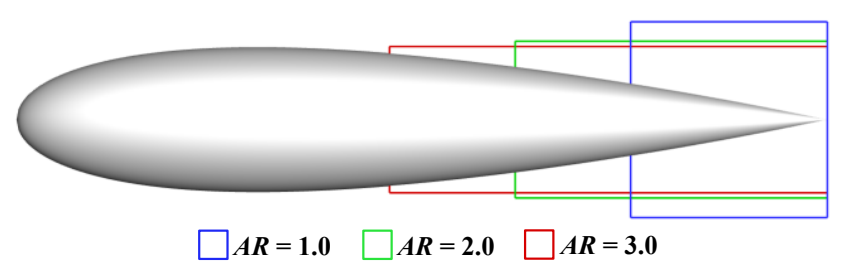

(b) Rectangle fin fold for deferent aspect ratio $A R: A R=$ $1.0,2.0$ and 3.0

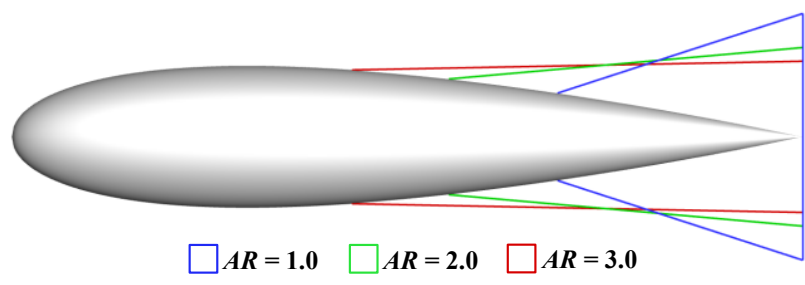

(c) Trapezoid fin fold for deferent aspect ratio $A R: A R=$ 1.0, 2.0 and 3.0.

Fig. 1 Front and bird's eye views for the fish body and description of two types of fin folds. 
The fish swimming motion in the $y$ direction was expressed by Eq. (2), where $L$ is the fish length, $T$ is the period and $A(t)$ is the amplitude function.

$$
y(t, x)=A(t)\left(\frac{x}{L}\right)^{2} \sin \left\{2 \pi\left(\frac{x}{L}-\frac{t}{T}\right)\right\}
$$

In order to avoid unstable swimming motions of the fish, amplitude function $A(t)$ was gradually increased from 0 to maximum amplitude $a_{a m p}$ within 8 cycles from the start of simulation to slowly develop the initial flow field. The first and second order derivatives of amplitude function $A(t)$ were set zero at $t=0,8 T$.

$$
A(t)=\left\{\begin{array}{cc}
a_{a m p}\left\{\frac{t}{8 T}-\frac{1}{2 \pi} \sin \left(\frac{\pi t}{4 T}\right)\right\} & (t<8 T) \\
a_{a m p} & (t \geq 8 T)
\end{array} .\right.
$$

In this simulation, the maximum amplitude $a_{a m p}$ was set $0.15 \mathrm{~L}$ (Li et al., 2012; Liu et al., 2017; Cui et al., 2018). Owing to viscous stress and pressure acting on the surface from the surrounding fluid, fish model moved in the $x$-axis and $y$-axis directions and rotated around the yaw-axis. These equations of motion were solved numerically by a third-order Adams-Bashforth method. Furthermore, Reynolds number $R e$ in the swimming simulation was defined by following equation:

$$
R e=\frac{U L}{v}
$$

where $U$ is the average swing speed at tail end and $v$ is the fluid kinematic viscosity. In this paper, $L, U$ and $R e$ were set $3.8 \mathrm{~mm}, 26.3 \mathrm{~mm} / \mathrm{s}$ and 100, respectively. Accordingly, the period $T$ was set $0.082 \mathrm{~s}$.

\subsection{Regularized lattice Boltzmann method}

The regularized lattice Boltzmann method (RLBM) (Izham et al., 2011; Morinishi and Fukui, 2016) was used as the governing equation of the fluid. The RLBM is designed to reduce calculation cost with modifying an algorithm in lattice Boltzmann method (LBM). In LBM, the distribution function $f_{\alpha}$ can be written using polynomial expression, as follows:

$$
f_{\alpha}=w_{\alpha}\left(a_{0}+b_{i} e_{\alpha i}+c_{i j} e_{\alpha i} e_{\alpha j}+\cdots\right)
$$

where $w_{\alpha}$ is the weight factor, $\boldsymbol{e}_{\boldsymbol{\alpha}}$ is the discrete velocity vector, and $a_{0}, b_{i}$ and $c_{i j}$ are the constant parameters with components of $i$ and $j$. The moments are used up to second order in the RLBM because it is equivalent to obtain second order accuracy for incompressible Navier-Stokes equation. Thus, the distribution function $f_{\alpha}$ is rewritten as

$$
f_{\alpha} \approx w_{\alpha}\left(a_{0}+b_{i} e_{\alpha i}+c_{i j} e_{\alpha i} e_{\alpha j}\right)
$$

In this report, we used 3D27V lattice speed model. Therefore, the weight factor $w_{\alpha}$ and the discrete velocity vector $\boldsymbol{e}_{\boldsymbol{\alpha}}$ were given by

$$
w_{\alpha}=\left\{\begin{array}{cc}
8 / 27 & (\alpha=0) \\
2 / 27 & (\alpha=1 \sim 6) \\
1 / 54 & (\alpha=7 \sim 18) \\
1 / 216 & (\alpha=19 \sim 26)
\end{array}\right.
$$




$$
\boldsymbol{e}_{\alpha}=\left\{\begin{array}{cc}
c(0,0,0) & (\alpha=0) \\
c( \pm 1,0,0), c(0, \pm 1,0), c(0,0, \pm 1) & (\alpha=1 \sim 6) \\
c( \pm 1, \pm 1,0), c( \pm 1,0, \pm 1), c(0, \pm 1, \pm 1) & (\alpha=7 \sim 18) \\
c( \pm 1, \pm 1, \pm 1) & (\alpha=19 \sim 26)
\end{array}\right.
$$

where $c$ is the speed of advection. Then, the fluid density $\rho$, the fluid momentum $\rho \boldsymbol{u}$, and non-equilibrium stress tensor $\Pi_{i j}{ }^{\text {neq }}$ are given by

$$
\begin{aligned}
& \rho=\sum_{\alpha} f_{\alpha}, \\
& \rho \boldsymbol{u}=\sum_{\alpha} f_{\alpha} \boldsymbol{e}_{\alpha}, \\
& \rho c_{s}^{2} \delta_{i j}+\rho u_{i} u_{j}+\Pi_{i j}^{n e q}=\sum_{\alpha} e_{\alpha i} e_{\alpha j} f_{\alpha},
\end{aligned}
$$

where $c_{s}$ is speed of sound and $\delta_{i j}$ is Kronecker delta. From Eqs. (9), (10) and (11), $a_{0}, b_{i}$ and $c_{i j}$ in Eq. (6) can be represented by physical quantities of the fluid. Then, Eq. (6) becomes

$$
f_{\alpha}=f_{\alpha}^{e q}+f_{\alpha}^{n e q}
$$

where $f_{\alpha}^{e q}$ is the equilibrium distribution function and $f_{\alpha}^{n e q}$ is the non-equilibrium distribution function, which can be expressed as follows, respectively

$$
\begin{aligned}
& f_{\alpha}^{e q}=w_{\alpha} \rho\left\{1+\frac{3\left(\boldsymbol{e}_{\alpha} \cdot \boldsymbol{u}\right)}{c^{2}}+\frac{9\left(\boldsymbol{e}_{\alpha} \cdot \boldsymbol{u}\right)^{2}}{2 c^{4}}-\frac{3 \boldsymbol{u}^{2}}{2 c^{2}}\right\} \\
& f_{\alpha}^{n e q}=\frac{9 w_{\alpha}}{2 c^{2}}\left(\frac{e_{\alpha i} e_{\alpha j}}{c^{2}}-\frac{1}{3} \delta_{i j}\right) \Pi_{i j}^{n e q} .
\end{aligned}
$$

Consequently, the time evolution of the distribution function $f_{\alpha}$ is given by

$$
f_{\alpha}\left(t+\delta t, \boldsymbol{x}+\boldsymbol{e}_{\alpha} \delta t\right)=f_{\alpha}(t, \boldsymbol{x})+\left(1-\frac{1}{\tau}\right) f_{\alpha}^{n e q}(t, \boldsymbol{x})
$$

In this study, the working fluid was assumed to be an incompressible fluid, and the pressure distribution function $p_{\alpha}$ was considered (He and Luo, 1997). The pressure distribution function $p_{\alpha}$ is defined by

$$
p_{\alpha}=c_{s}^{2} f_{\alpha}
$$

Using Eq. (16), Eqs. (13) and (15) can be represented as follows:

$$
\begin{aligned}
& p_{\alpha}^{e q}=w_{\alpha}\left[p+\rho_{0}\left\{\left(\boldsymbol{e}_{\alpha} \cdot \boldsymbol{u}\right)+\frac{3\left(\boldsymbol{e}_{\alpha} \cdot \boldsymbol{u}\right)^{2}}{2 c^{2}}-\frac{\boldsymbol{u}^{2}}{2}\right\}\right] \\
& p_{\alpha}\left(t+\delta t, \boldsymbol{x}+\boldsymbol{e}_{\alpha} \delta t\right)=p_{\alpha}(t, \boldsymbol{x})+\left(1-\frac{1}{\tau}\right) p_{\alpha}^{n e q}(t, \boldsymbol{x}),
\end{aligned}
$$

where $\rho_{0}$ is the fluid reference density. Accordingly, the pressure $p$ and fluid velocity $\boldsymbol{u}$ are given by 


$$
\begin{aligned}
& p=\sum_{\alpha} p_{\alpha}, \\
& \boldsymbol{u}=\frac{1}{\rho_{0} c_{s}^{2}} \sum_{\alpha} p_{\alpha} \boldsymbol{e}_{\alpha} .
\end{aligned}
$$

\subsection{Virtual flux method}

The virtual flux method (Tanno et al., 2006; Morinishi and Fukui, 2012) was used to calculate interfaces between fluid and fish model on a Cartesian grid. This method has advantages to capture pressure fields around the object sharply.

Figure 2 shows a two-dimensional schematic view of the virtual boundary points. The virtual boundary points are provided as the points where the virtual surfaces cross the lattice or diagonal lines of the grid points. At the virtual boundary points, the virtual flux satisfies the following boundary conditions.

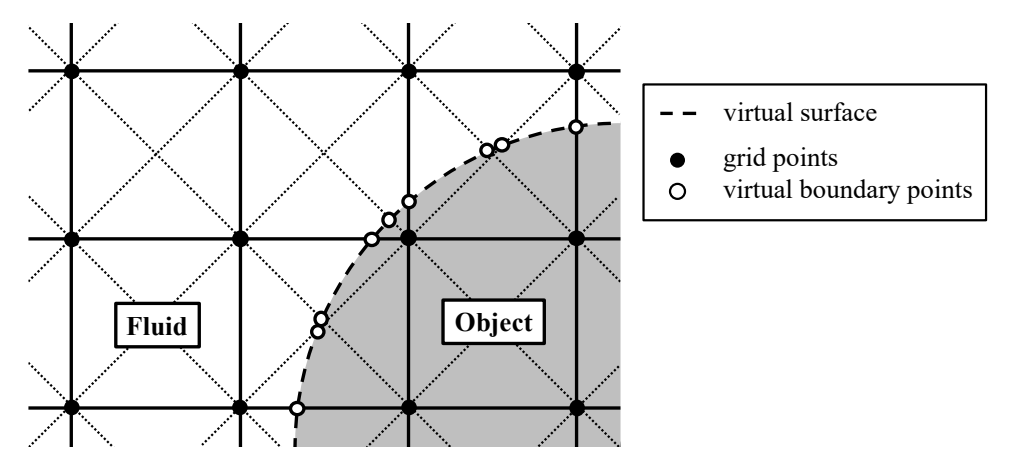

Fig. 2 Schematic view of virtual boundary points.

No-slip boundary conditions were applied at the virtual boundary points, and the virtual boundary velocity $\boldsymbol{u}_{\mathbf{v b}}$ and pressure $p_{\mathrm{vb}}$ were given by

$$
\begin{aligned}
& \boldsymbol{u}_{\mathrm{vb}}=\boldsymbol{u}_{\mathrm{wall}}, \\
& \left.\frac{\partial p}{\partial n}\right|_{\mathrm{vb}}=0,
\end{aligned}
$$

where $\boldsymbol{u}_{\text {wall }}$ is the object velocity at the virtual boundary points and $\boldsymbol{n}$ is the unit normal vector on the object surface. Eq. (22) can be rewritten using Taylor expansion, as follow:

$$
p_{\mathrm{vb}}=\frac{h_{2}{ }^{2} p_{1}-h_{1}{ }^{2} p_{2}}{h_{2}{ }^{2}-h_{1}{ }^{2}} .
$$

In the above equation, $p_{1}$ and $p_{2}$ are the pressures at the points that are $h_{1}$ and $h_{2}$ away from the object surface in the normal direction, respectively. In this study, $h_{1}=\sqrt{3} \delta x$ and $h_{2}=2 \sqrt{3} \delta x$ were applied for three-dimensional analysis.

Figure 3 shows a flow field in which a fluid and an object are separated by the virtual boundary. 


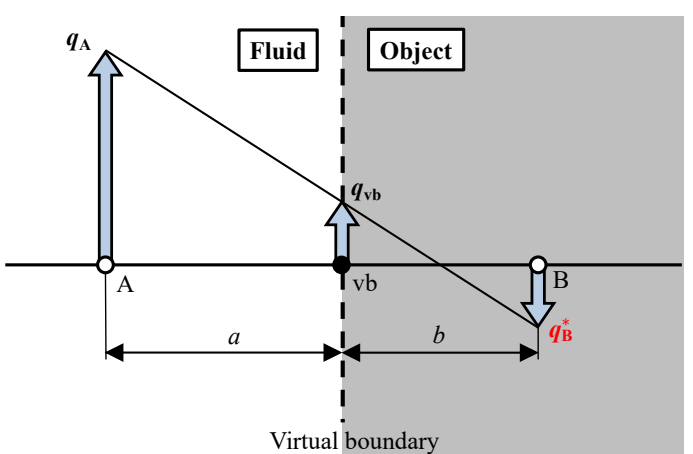

(a) $a \geq 0.5$

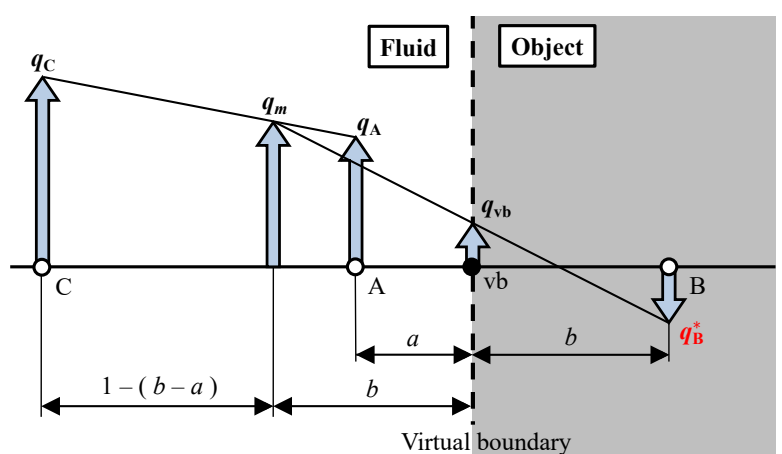

(b) $a<0.5$

Fig. 3 Extrapolation of physical quantity $\boldsymbol{q}$, where $a$ and $b$ are internal ratios of the line AB. These internal ratios satisfy a condition: $a+b=1$.

The pressure distribution function at the grid point A propagates from the pressure distribution function at the grid point B. However, the particle at the grid point B is unable to travel across the virtual surface toward the grid point A and is reflected at the virtual boundary. Therefore, the virtual pressure distribution function $p_{\alpha}^{*}$ at the point $\mathrm{B}$ is estimated from the boundary conditions as shown in Eqs. (21) and (23). The virtual pressure distribution function $p_{\alpha}^{*}$ can be obtained from the virtual physical quantity $q_{\mathrm{B}}^{*}$ which is calculated by linear extrapolation. In the case of $a \geq 0.5$, the virtual physical quantity $q_{\mathrm{B}}^{*}$ was given by Eq. (24). Conversely, when internal ratio $a$ is small, the calculation becomes unstable due to the denominator of the right-hand side of Eq. (24). Then the physical quantity at the point that is $b \delta x$ away from the virtual boundary point $\mathrm{vb}$ was interpolated from the grid points $\mathrm{A}$ and $\mathrm{C}$, and $q_{\mathrm{B}}^{*}$ was given by

$$
\begin{aligned}
& q_{\mathrm{B}}^{*}=\frac{b}{a} q_{\mathrm{A}}+\frac{1}{a} q_{\mathrm{vb}} \quad(a \geq 0.5), \\
& q_{\mathrm{B}}^{*}=2 q_{\mathrm{vb}}-\left[(b-a) q_{\mathrm{C}}+\{1-(b-a)\} q_{\mathrm{A}}\right]=2 q_{\mathrm{vb}}-q_{m} \quad(a<0.5) .
\end{aligned}
$$

Substituting the virtual physical quantity $q_{\mathrm{B}}^{*}$ into Eq. (17), the virtual equilibrium pressure distribution function $p_{\alpha}^{e q^{*}}$ was given as follow:

$$
p_{\alpha}^{e q^{*}}\left(t, \boldsymbol{x}_{\mathbf{B}}\right)=w_{\alpha}\left[p_{\mathrm{B}}^{*}+\rho_{0}\left\{\left(\boldsymbol{e}_{\alpha} \cdot \boldsymbol{u}_{\mathbf{B}}^{*}\right)+\frac{3\left(\boldsymbol{e}_{\alpha} \cdot \boldsymbol{u}_{\mathbf{B}}^{*}\right)^{2}}{2 c^{2}}-\frac{\boldsymbol{u}_{\mathbf{B}}^{* 2}}{2}\right\}\right] .
$$

Furthermore, the virtual non-equilibrium pressure distribution function $p_{a}^{\text {neq* }}$ was given by approximate nearest neighbor as follows:

$$
\begin{aligned}
& p_{\alpha}^{n e q^{*}}\left(t, x_{\mathbf{B}}\right)=p_{\alpha}^{n e q}\left(t, x_{\mathbf{A}}\right) \quad(a \geq 0.5), \\
& p_{\alpha}^{n e q^{*}}\left(t, x_{\mathbf{B}}\right)=\left[(b-a) p_{\alpha}^{n e q}\left(t, x_{\mathbf{C}}\right)+\{1-(b-a)\} p_{\alpha}^{n e q}\left(t, x_{\mathbf{A}}\right)\right] \quad(a<0.5) .
\end{aligned}
$$

Thus, the pressure distribution function in the next timestep was obtained from Eq. (29) by the virtual equilibrium pressure distribution function $p_{\alpha}^{e q^{*}}$ and the virtual non-equilibrium pressure distribution function $p_{\alpha}^{n e q^{*}}$.

$$
p_{\alpha}\left(t+\delta t, \boldsymbol{x}_{\mathbf{A}}\right)=p_{\alpha}^{e q^{*}}\left(t, \boldsymbol{x}_{\mathbf{B}}\right)+\left(1-\frac{1}{\tau}\right) p_{\alpha}^{n e q^{*}}\left(t, \boldsymbol{x}_{\mathbf{B}}\right) .
$$

This method also can be applied to shell elements without thickness, such as fish larvae's fin fold. Note that since fish 
body moves dynamically in the Cartesian coordinate system, some procedures are necessary to modify the flow field around the body surface in case a grid occupied by the fish body goes outside in the next timestep. In such a situation, linear extrapolations of the distribution function are employed. Distribution functions at two points with distances of $\sqrt{3} \delta x$ and $2 \sqrt{3} \delta x$ from the body surface in the normal direction are utilized for the extrapolations. These distribution functions are also obtained by interpolating those of surrounding 8 grid points. Flow fields around the body surface with these procedures have been validated with data from others (Trizila et al., 2011; Wang and He, 2015).

\subsection{Simulation setups and verification}

In this paper, we investigated the effects of aspect ratio of fish larvae's fin fold on its propulsion performance by fish swimming simulations.

Figures 4 and 5 show the schematic view of the computational domain and mesh. 4-tiered multi block method (Yu et al., 2002; Yu and Girimaji, 2006; Aroa et al., 2016) was employed and the calculation domain was then divided into 4 blocks with different grid resolutions. The inner three blocks moved in conjunction with the fish model. The calculation domain has the size of $20 L \times 10 L \times 5 L$. The symmetric boundary condition was applied to the bottom boundary, and the outflow boundary condition was applied to the other boundaries. As an initial condition, fish model was placed at position $(9 L, 5 L, 0)$. Then the fish model's motion due to hydrodynamic forces acting on the model's surface was numerically obtained using Newton's second law of motion (Eq. (30)) and Euler's equation of motion (Eq. (31)):

$$
\begin{aligned}
& m \frac{d^{2} \boldsymbol{x}}{d t^{2}}=\boldsymbol{F} \\
& I \frac{d^{2} \theta}{d t^{2}}=T q,
\end{aligned}
$$

where $m$ is the mass, $\boldsymbol{x}$ is the position vector of the center of gravity, $\boldsymbol{F}$ is the hydrodynamic forces acting on the surface, $I$ is the moment of inertia, $\theta$ is the angle, and $T q$ is the torque. These equations were discretized by a third-order Adams-Bashforth method and interactively solved between fluid and fish model.

Then the propulsion performance was evaluated by swimming speed $U_{S W}$, thrust force $F_{T}$, power requirement $P$ and swimming efficiency $\eta\left(\eta=\overline{U_{S W}} \cdot \overline{F_{T}} / \bar{P}\right)$, where thrust force is summation of fluid forces acting only in the $x$-axis negative direction. Note that thrust force was defined as not net thrust force but relative force to the swimming direction referred to the study by Li et al. (2012).

$$
F_{T}=\sum_{l}^{\text {surface }} \frac{1}{2}\left(\left|F_{x, l}\right|-F_{x, l}\right)
$$

where $F_{x, l}$ is the force component in the propulsion direction at a surface element $l$ of the fish model. And the power requirement $P$ was defined as summation of hydrodynamic power $P_{\text {hydro }}$ and inertia power $P_{\text {ineartia: }}$ :

$$
P=P_{\text {hydro }}+P_{\text {inertia }}
$$

The hydrodynamic power $P_{\text {hydro }}$ was obtained using velocity vector $\boldsymbol{u}_{l}$ at a surface element $l$ of the fish model:

$$
P_{\text {hydro }}=\sum_{l}^{\text {surface }} \boldsymbol{u}_{l} \cdot\left(-\boldsymbol{F}_{l}\right) \text {, }
$$

where $\boldsymbol{F}_{l}$ is the hydrodynamic force acting on the element $l$. The inertia power $P_{\text {ineartia }}$ was obtained using center of gravity $\left(x_{n}, y_{n}, z_{n}\right)$ of a tetrahedral element $n$ : 


$$
P_{\text {inertia }}=\sum_{n}^{\text {body }}\left(\left|\frac{d x_{n}}{d t} \cdot \frac{d^{2} x_{n}}{d t^{2}}\right|+\left|\frac{d y_{n}}{d t} \cdot \frac{d^{2} y_{n}}{d t^{2}}\right|+\left|\frac{d z_{n}}{d t} \cdot \frac{d^{2} z_{n}}{d t^{2}}\right|\right) \Delta m_{n}
$$

where $\Delta m_{n}$ is the mass of the tetrahedral element $n$.

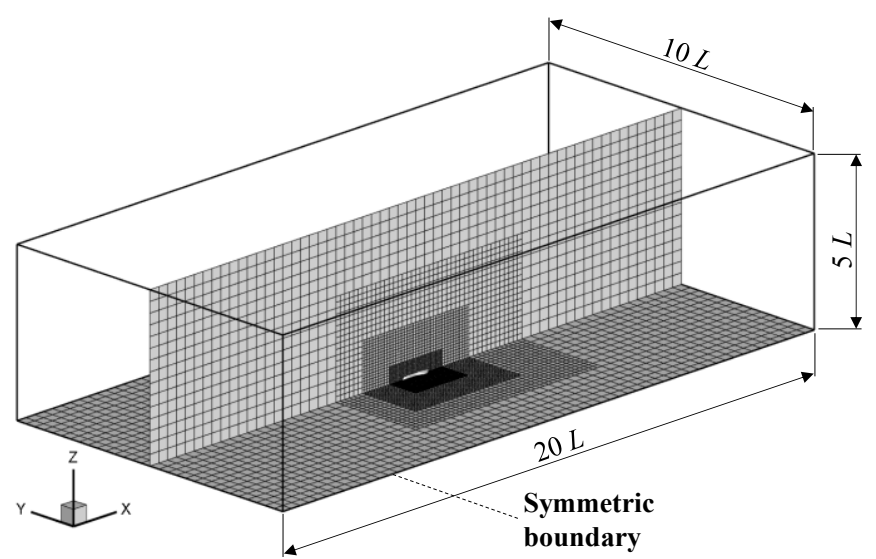

Fig. 4 Schematic view of the computational domain for bird's eye view.

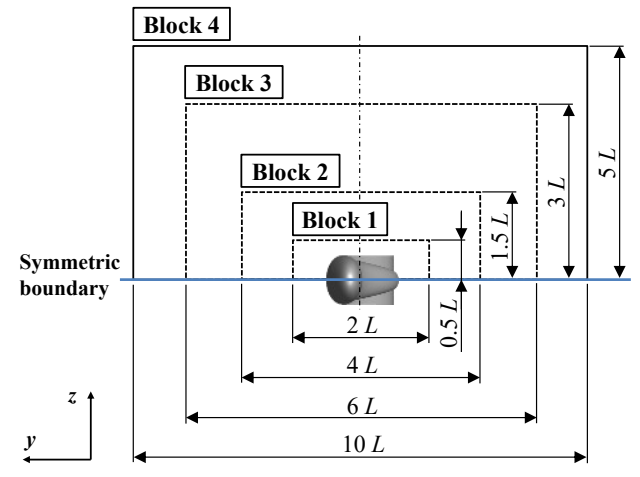

(a) $y-z$ plane.

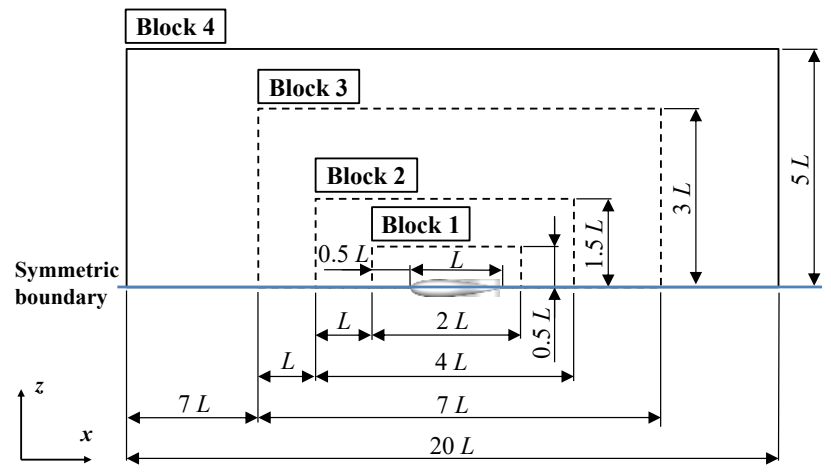

(b) $x-z$ plane.

Fig. 5 Schematic view of the computational domain for two dimensional views.

A verification study was conducted for the normal fish model as shown in Fig. 1(a). An independence study of a number of grids in characteristic length $N_{L}$ was tested by swimming speed $U_{S W}$. This test was conducted with four different numbers of grids in characteristic length $N_{L}: N_{L}=112,160,224$, and 320. Fish models swam 30 cycles in total, and averaged swimming speed $\overline{U_{S W}}$ in the last four cycles was evaluated. Figure 6 shows the time history of the swimming speed $U_{S W}$ in the last cycle and the comparison of four-cycle averaged values. The time history of the swimming speed $U_{S W}$ shows a similar trend among different grid resolutions, and the values become smaller as the number of grids in characteristic length $N_{L}$ increases. Table 1 shows the four-cycle averaged swimming speed $\overline{U_{S W}}$ and the error from convergence estimation, where convergence estimation is obtained by the approximation curve in Fig. 6(b). In the case of $N_{L}=320$, the error is as small as $0.7744 \%$, and this grid resolution is appropriate to consider the effects of aspect ratio $A R$ of fin fold. Hence, the number of grids in characteristic length in this research is set to $N_{L}=320$. 


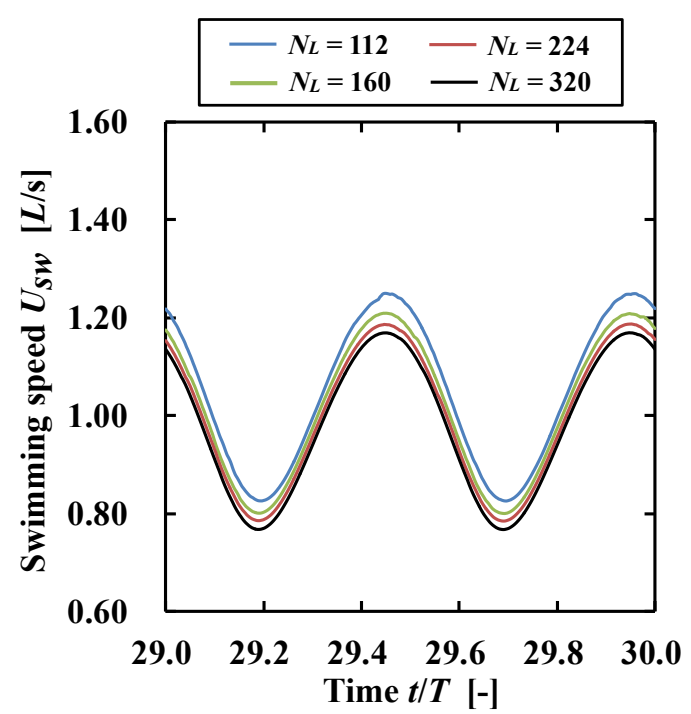

(a) Time history of swimming speed $U_{S W}$ in the 30th period for four number of grids $N_{L}: N_{L}=112,160,224$ and 320 .

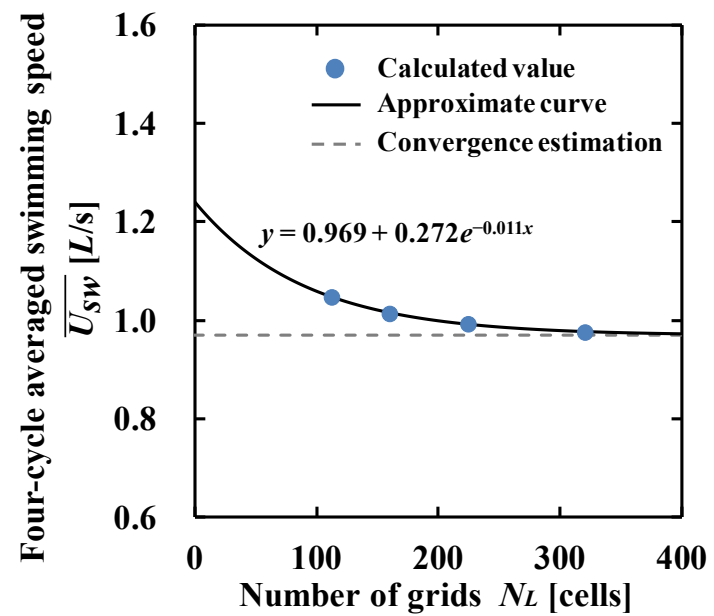

(b) Relationship between four-cycle averaged swimming speed $\overline{U_{S W}}$ and number of grids $N_{L}$, and approximate curve from four calculated values.

Fig. 6 Grid independence study for the flow around small swimming object.

Table 1 Four-cycle averaged swimming speed for different number of grids in characteristic length.

\begin{tabular}{ccc}
\hline \hline$N_{L}$ & $\overline{U_{S W}}[L / \mathrm{s}]$ & Error [\%] \\
\hline 112 & 1.048 & 8.148 \\
\hline 160 & 1.015 & 4.691 \\
\hline 224 & 0.9930 & 2.456 \\
\hline 320 & 0.9767 & 0.7744 \\
\hline Convergence estimation & 0.9692 & - \\
\hline
\end{tabular}

\section{Results and Discussion}

Based on the model shown in Fig. 1(a), aspect ratio $A R$ of 1.0 to 3.5 in 0.5 steps under constant surface area condition were studied. As with the verification study in section 2.3, fish models swam 30 cycles in total, and physical quantities in the last 4 cycles were evaluated as propulsion performance.

Figure 7 shows the relationship of propulsion performance with aspect ratio $A R$. As shown in Fig. 7(a), averaged thrust force $\overline{F_{T}}$ monotonically decreases as aspect ratio $A R$ becomes high. This is because lower aspect ratio $A R$ models produce stronger thrust force during one stroke by the wider fin fold near the tail end. When the thickness and density are assumed to be constant, the inertia moment $I$ of the fin fold becomes higher for lower $A R$ condition. Therefore, the thrust force $F_{T}$ would be proportional to the inertia moment $I$ as mentioned previously by Kikuchi et al (2014). Averaged swimming speed $\overline{U_{S W}}$ reaches a peak at $A R=2.5$ in the rectangle fin fold model. Lower aspect ratio $A R$ models produce less thrust force at the center of the fish body due to the shorter fin fold, and the amplitude of swimming speed $A_{U_{S W}}$ divided by its average $\overline{U_{S W}}$ is larger for $A R=1.0$ as shown in Table 2 . Hence, averaged swimming speed $\overline{U_{S W}}$ is low at low aspect ratio $A R$ due to wasting energy for acceleration and deceleration. In Fig. 7(b), averaged power requirement $\bar{P}$ has a similar tendency for that of averaged thrust force $\overline{F_{T}}$ because of higher power expenditure near the tail end. Swimming efficiency $\eta$ tendency is almost similar to that of swimming speed. In the rectangle fin fold model, swimming efficiency $\eta \cdot$ had a peak with respect to aspect ratio $A R$, and it obtained maximum value at $A R=2.5$. When the aspect ratio $A R$ is high, no significant difference is found for four values in Fig. 7 between two types of fin folds due to similarity of the fin fold shape. 


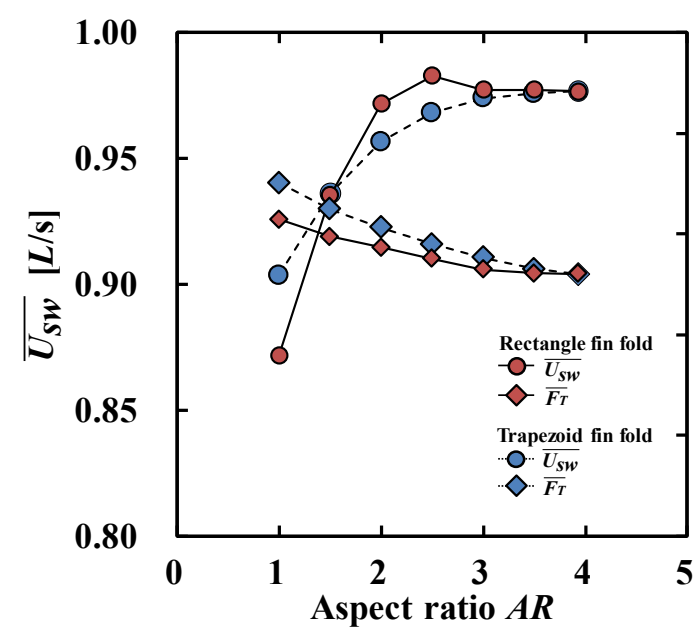

(a) Swimming speed $U_{S W}$ and thrust force $F_{T}$.

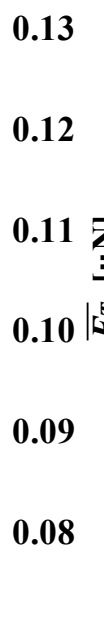

Fig. 7 Four four-cycle averaged values versus aspect ratio $A R$ for two types of fin folds. Different markers indicate different evaluation values, and red and blue colors correspond to rectangle and trapezoid fin folds, respectively.

Table 2 Four-cycle averaged swimming speed $\overline{U_{S W}}$ and its normalized amplitude $A_{U_{S W}} / \overline{U_{S W}}$.

\begin{tabular}{ccccc}
\hline \multirow{2}{*}{$A R$} & \multicolumn{2}{c}{ Rectangle fin fold } & \multicolumn{2}{c}{ Trapezoid fin fold } \\
\cline { 2 - 5 } & $\overline{U_{S W}}[\mathrm{~L} / \mathrm{s}]$ & $A_{U_{S W}} / \overline{U_{S W}}[-]$ & $\overline{U_{S W}}[\mathrm{~L} / \mathrm{s}]$ & $A_{U_{S W}} / \overline{U_{S W}}[-]$ \\
\hline 1.0 & 0.8735 & 0.2627 & 0.9035 & 0.2786 \\
\hline 1.5 & 0.9354 & 0.2188 & 0.9361 & 0.2454 \\
\hline 2.0 & 0.9718 & 0.2124 & 0.9566 & 0.2290 \\
\hline 2.5 & 0.9829 & 0.2112 & 0.9682 & 0.2196 \\
\hline 3.0 & 0.9773 & 0.2088 & 0.9739 & 0.2139 \\
\hline 3.5 & 0.9772 & 0.2075 & 0.9759 & 0.2095 \\
\hline 3.93 & 0.9767 & 0.2075 & 0.9767 & 0.2075 \\
\hline
\end{tabular}

Figures 8 and 9 show the vortex visualization and pressure distribution on the fish surface at $t=29.35 T$. Note that $Q^{*}$ in Figs. 8 and 9 indicates nondimensional second invariant of the velocity gradient tensor $Q$ :

$$
\begin{aligned}
& Q=\frac{1}{2}\left(W_{i j} W_{i j}-S_{i j} S_{i j}\right), \\
& Q^{*}=\frac{Q}{(U / L)^{2}},
\end{aligned}
$$

where $W_{i j}$ is the rate of rotation tensor, and $S_{i j}$ is the rate of deformation tensor. According to the vortex structure at $A R$ $=2.5$ in Fig. 8(a), vortices were formed from the head tip to the tail end of the fish model. The vortices were generated at the head tip and traveled backward along the fish body and fin fold. These vortices shed when the body axis reached the lateral extreme of the body wave or when they reached the tail end. Near the center of the fish body and the tail end, there were two pairs of vortices rotating in the opposite direction up and down. As shown in Fig. 8(b), the pressure difference was large where the vortices pairs were located, which is in accordance with the results in Li et al. (2016) of swimming simulation. Thus, the edge vortices along the fin fold generate thrust force due to the large pressure difference between the front and back sides of the fin fold. Comparing the rectangle and trapezoid fin fold models at $A R=2.5$, the vortex structures are similar (Fig. 8(a) and (c)). Although it also can be seen similar pressure distribution at the surface of the fish models (Fig. 8(b) and (d)), in the trapezoid fin fold model, the contribution of generating thrust force by the anterior edge vortices pair is smaller because of the narrower fin fold at the center of the model. On the other hand, according to the vortex structure at $A R=1.0$ in Fig. 9(a), there was only one pair of vortices at the fin fold edge because of the shorter fin fold. The pressure difference was not so large on the anterior parts of the fin fold in Fig. 9(b). Thus, lower aspect ratio $A R$ models produce less thrust force at the center of the fish body because of the 
undeveloped edge vortices, and this leads to large amplitude of its swimming speed as shown in Table 2. Regarding the trapezoid fin fold model in Fig. 9(c) and (d), a weak edge vortex formed at the center of the fish body. However, the large pressure difference area at the anterior parts of the fin fold was small, and it did not generate enough thrust force to stabilize the swimming speed.

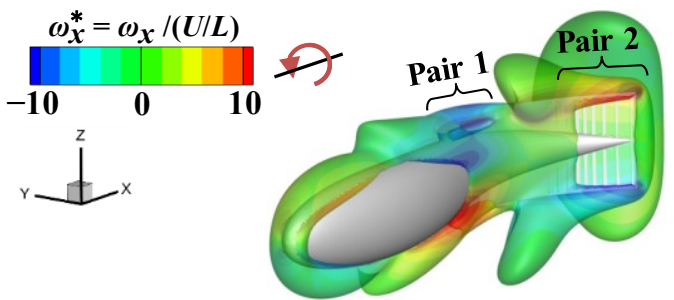

(a) Vortex structure for rectangle fin fold.

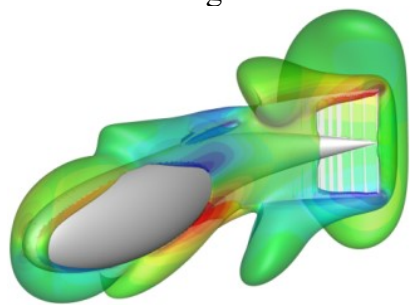

(c) Vortex structure for trapezoid fin fold.

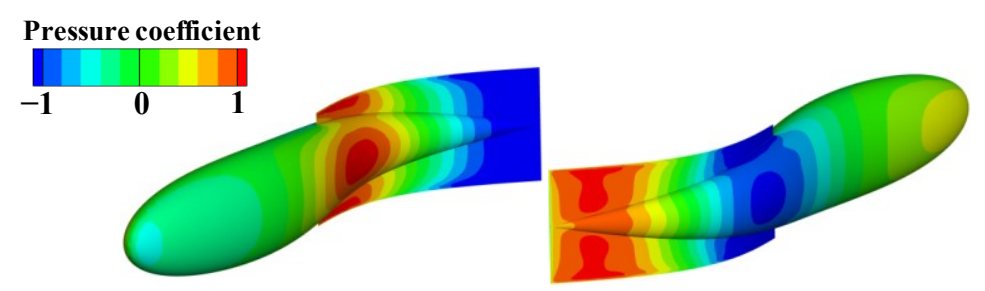

(b) Pressure distribution for rectangle fin fold.
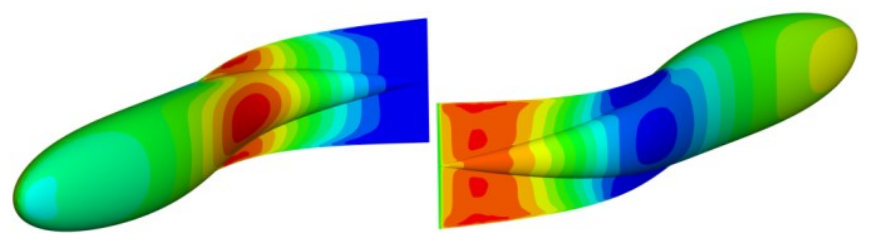

(d) Pressure distribution for trapezoid fin fold.

Fig. 8 Isosurface of $Q^{*}=0.05$ colored by the vorticity around the $x$-axis $\omega_{x}^{*}$ and pressure distribution on the fish surface at $t=29.35 T$ for two types of fin folds at $A R=2.5$.

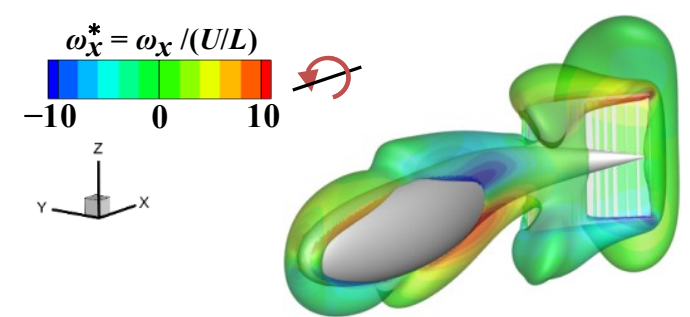

(a) Vortex structure for rectangle fin fold.

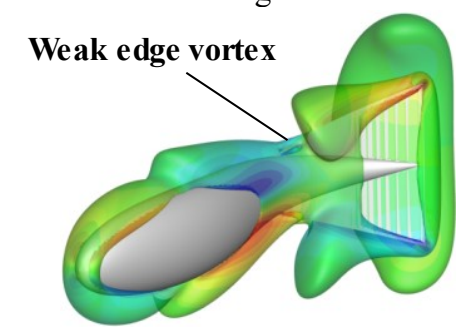

(c) Vortex structure for trapezoid fin fold.

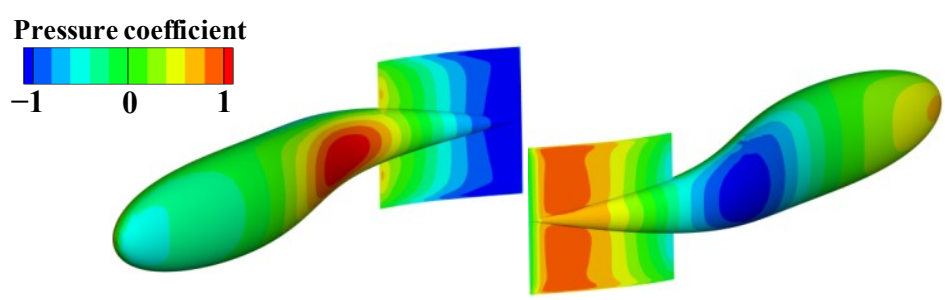

(b) Pressure distribution for rectangle fin fold.

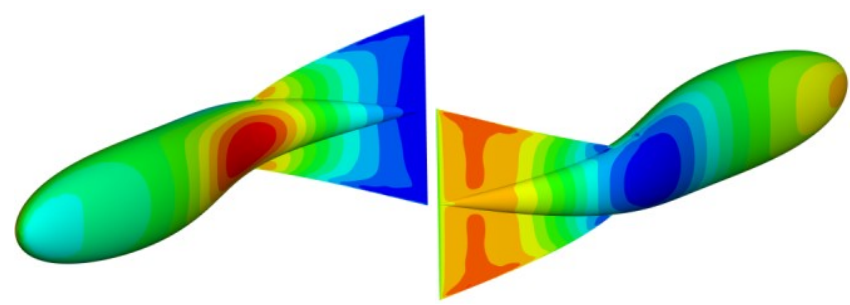

(d) Pressure distribution for trapezoid fin fold.

Fig. 9 Isosurface of $Q^{*}=0.05$ colored by the vorticity around the $x$-axis $\omega_{x}^{*}$ and pressure distribution on the fish surface at $t=29.35 T$ for two types of fin folds at $A R=1.0$.

Figure 10 shows the distribution of one-cycle averaged fluid force acting in the propulsive direction ( $x$-axis negative direction) on the fish surface in the case of $A R=1.0$ and 2.5. Positive fluid force was generated along the fin fold, and these areas corresponded to the position where strong edge vortices occurred. In particular, large thrust force was produced near the tail end, where the swimming motion amplitude is large. Lower aspect ratio $A R$ models generated less thrust force at the center of the fish model than higher aspect ratio $A R$ models.

The present result suggested that lower aspect ratio $A R$ models with wider fin fold produce stronger thrust force during one tail end stroke. Conversely, higher aspect ratio $A R$ models with longer fin fold produce thrust force in a wider part of the fish body. This may lead to stabilizing swimming motions by generating thrust force at the timing different from a tail end stroke during a cycle. 
It should be also noted that in this study, thrust force was defined as not net thrust force but relative force to the swimming direction. This thrust force could not evaluate swimming stability in the higher aspect ratio $A R$ models without considering drag force. Hence, to describe relation between swimming stability and fluid force, alternative evaluation index should be considered in future work.

One of the novelties of this study is to seek the effective shape of the fin fold according to its growth stage. The shapes of the fin fold were changed under the same area condition including rectangle and trapezoid. Both fin fold models with the aspect ratio $A R=2.5$ and more showed better performance, which indicates these shapes would be optimized under the Reynolds number $R e=100$. These findings are consistent with the actual fin folds of the fish larvae: fin fold shapes of the fish larvae are optimized to some extent. On the other hand, since fin fold shapes and Reynolds number are changed with their growth stages, a hypothesis can be made; if their fin fold shapes might be optimized to some extent depending on their growth stages, the aspect ratio $A R$ for their best performance could be decreased with increasing $R e$. These relationships between $A R$ and $R e$ should be considered in detail. Although this research is still fundamental, these numerical models proposed here are promising for further discussion in the future.

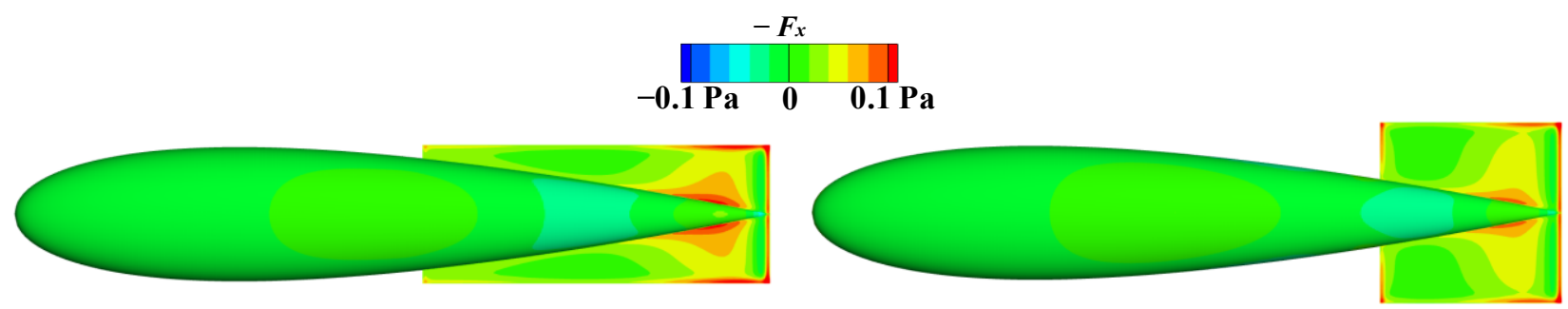

(a)Rectangle fin fold at $A R=2.5$

(b) Rectangle fin fold at $A R=1.0$

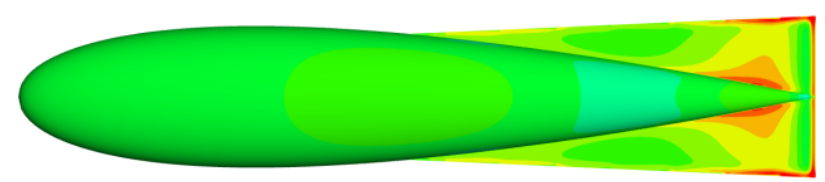

(c)Trapezoid fin fold at $A R=2.5$

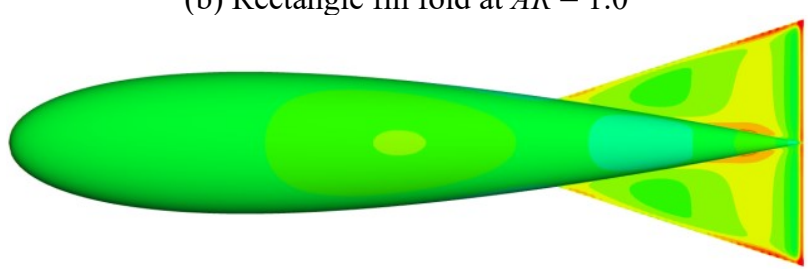

(d)Trapezoid fin fold at $A R=1.0$

Fig. 10 One-cycle averaged fluid force in negative- $x$-direction in per unit area distribution on the fish surface for two types of fin folds.

\section{Conclusions}

In this research, swimming simulation was performed with focusing on fish larvae to investigate the effects of aspect ratio of two types of fin folds on its propulsion performance. Based on the results in this paper, the following conclusions have been drawn.

(1) The edge vortices traveling backward along the fin fold contribute to the generating thrust force by increasing the pressure difference between the front and back sides of the fin fold.

(2) When aspect ratio $A R$ of fin fold is high, fish models produce thrust force in a wider part of the fish body with two pairs of vortices and swim efficiently by stabilizing its swimming speed.

(3) In the case of the same aspect ratio of fin fold, trapezoid fin fold model generates less thrust force at the center of the body than rectangle fin fold model because of the narrower fin fold at the area. In trapezoid fin fold model, thrust force generated near the tail end is larger, but swimming amplitude is also larger, resulting in the lower swimming efficiency.

(4) Fish living in the low Reynolds number regime such as $R e=100$ are subjected to large viscous force from the fluid. They are strongly decelerated periodically between acceleration periods by the tail end stroke. Therefore, it is important for the swimming mechanism to generate a large thrust force by the tail end and to stabilize the swimming speed by the other parts of body. 


\section{References}

Aritani, T., Kawasaki, N. and Takada, Y., Small Robotic Fish with Two Magnetic Actuators for Autonomous Tracking of a Goldfish, JOURNAL OF AERO AQUA BIO-MECHANISMS, Vol.8, No.1 (2019), pp.69-74.

Arora, N., Gupta, A. and Shyy, W., A shifting discontinuous-grid-block lattice Boltzmann method for moving boundary simulations, Computers and Fluids, Vol.125 (2016), pp.59-70.

Cui, Z., Yang, Z., Shen, L. and Jiang, H. Z., Complex modal analysis of the movements of swimming fish propelled by body and/or caudal fin, Wave Motion, Vol.78 (2018), pp.83-97.

He, X. and Luo, L. S., Lattice Boltzmann model for the incompressible Navier-Stokes equation, Journal of Statistical Physics, Vol.88 (1997), pp.927-944.

Izham, M., Fukui, T. and Morinishi, K., Application of regularized lattice Boltzmann method for incompressible flow simulation at high Reynolds number and flow with curved boundary, Journal of Fluid Science and Technology, Vol.6, No.6 (2011), pp.812-822.

Kikuchi, K., Uehara, Y., Kubota, Y. \& Mochizuki, O., Morphological consideration of fish shape on thrust generation, Journal of Applied Fluid Mechanics, Vol.7 (2014), pp.625-632.

Li, G., Kolomenskiy, D., Liu, H., Thiria, B. and Godoy-Diana, R., On the energetics and stability of a minimal fish school, PLoS ONE, Vol.14, No.8 (2019), pp.1-20.

Li, G., Muller, U. K., Van Leeuwen, J. L. and Liu, H., Body dynamics and hydrodynamics of swimming fish larvae: a computational study, Journal of Experimental Biology, Vol.215, No.22 (2012), pp.4015-4033.

Li, G., Muller, U. K., Van Leeuwen, J. L. and Liu, H., Fish Larvae Exploit Edge Vortices along Their Dorsal and Ventral Fin Folds to Propel Themselves, Journal of The Royal Society Interface, Vol.13, No.116 (2016), pp.1-14.

Liu, G., Ren, Y., Dong, H., Akanyeti, O., Liao, J. C. and Lauder, G. V., Computational analysis of vortex dynamics and performance enhancement due to body-fin and fin-fin interactions in fish-like locomotion, Journal of Fluid Mechanics, Vol.829, No.25 (2017), pp.65-88.

Luo, Y., Xiao, Q., Shi, G., Wen, L., Chen, D. and Pan, G., A fluid-structure interaction solver for the study on a passively deformed fish fin with non-uniformly distributed stiffness, Journal of Fluids and Structures, Vol.92 (2020), pp.1-24.

Maki, T., AUV: Autonomous Underwater Vehicle, A Journal of the Japan Society of Mechanical Engineers, Vol.121, No.1199 (2018), pp.24-27 (in Japanese).

Mchenry, M. J. and Lauder, G. V., The mechanical scaling of coasting in zebrafish (Danio rerio), Journal of Experimental Biology, Vol.208, No.12 (2005), pp.2289-2301.

Morinishi, K. and Fukui, T., An Eulerian approach for fluid-structure interaction problems, Computers and Fluids, Vol.65 (2012), pp.92-98.

Morinishi, K. and Fukui, T., Parallel computation of turbulent flows using moment base lattice Boltzmann method, International Journal of Computational Fluid Dynamics, Vol.30, No.5 (2016), pp.363-369.

Ota, K., Nouda, S., Takeuchi, T., Iguchi, M., Kojima, Y., Kuramoto, T., Inoue, T., Shindo, Y., Uesugi, K., Fujito, Y., Nishihara, H., Ohtsuka, N., Higuchi, K. and Team Mermaid, What Kind of Capsule Endoscope Is Suitable for a Controllable Self-Propelling Capsule Endoscope? Experimental Study Using a Porcine Stomach Model for Clinical Application (with Videos), PLoS ONE, Vol.10, No.10 (2015), pp.1-11.

Tanno, I., Morinishi, K., Matsuno, K. and Nishida, H., Validation of virtual flux method for forced convection flow, JSME International Journal Series B: Fluids and Thermal Engineering, Vol.49, No.4 (2007), pp.1141-1148.

Trizila, P., Kang, C. K., Aono, H., Shyy, W. and Visbal, M., Low-reynolds-number aerodynamics of a flapping rigid flat plate, AIAA Journal, Vol.49, No.4 (2011), pp.806-823.

Wang, S. and He, G., Lift enhancement on spanwise oscillating flat-plates in low-reynolds-number flows, Physics of Fluids, Vol.27 (2015), pp.1-19.

Wei, Z., Jinxing, L.,Leong, Y. J.,Rozen, I., Qu, X., Dong, R., Wu, Z., Gao, W., Chung, P. H., Wang, J. and Chen, S., 3D-Printed Artificial Microfish, Advanced Materials, Vol.27, No.30 (2015), pp.4411-4417.

Yu, D. and Girimaji, S. S., Multi-block Lattice Boltzmann method: Extension to 3D and validation in turbulence, Physica A: Statistical Mechanics and its Applications, Vol.362, No.1 (2006), pp.118-124.

Yu, D., Mei, R. and Shyy, W., A multi-block lattice Boltzmann method for viscous fluid flows, International Journal for Numerical Methods in Fluids, Vol.39, No.2 (2002), pp.99-120. 
Bamba, Fukui and Morinishi, Journal of Fluid Science and Technology, Vol.16, No.3 (2021)

Zhu, J., White, C., Wainwright, D. K., Di Santo, V., Lauder, G. V. and Bart-Smith, H., Tuna robotics: A high-frequency experimental platform exploring the performance space of swimming fishes, Science Robotics, Vol.4, No.34 (2019), pp.1-12. 